\title{
Application of Super-resolution Image Reconstruction to Digital Holography
}

\author{
Shuqun Zhang \\ Department of Computer Science, College of Staten Island, City University of New York, 2800 Victory Boulevard, \\ Staten Island, NY 10314, USA
}

Received 1 December 2004; Revised 10 April 2005; Accepted 18 April 2005

\begin{abstract}
We describe a new application of super-resolution image reconstruction to digital holography which is a technique for threedimensional information recording and reconstruction. Digital holography has suffered from the low resolution of CCD sensors, which significantly limits the size of objects that can be recorded. The existing solution to this problem is to use optics to bandlimit the object to be recorded, which can cause the loss of details. Here super-resolution image reconstruction is proposed to be applied in enhancing the spatial resolution of digital holograms. By introducing a global camera translation before sampling, a highresolution hologram can be reconstructed from a set of undersampled hologram images. This permits the recording of larger objects and reduces the distance between the object and the hologram. Practical results from real and simulated holograms are presented to demonstrate the feasibility of the proposed technique.
\end{abstract}

Copyright @ 2006 Hindawi Publishing Corporation. All rights reserved.

\section{INTRODUCTION}

Due to the physical limitations and cost of imaging sensors, it is difficult to obtain high-resolution images in many applications. The low-resolution images are often degraded by noise, blurring, and aliasing effects. Super-resolution image reconstruction has been demonstrated to be very effective in removing these effects by combining several low-resolution images to yield a single high-resolution image. Numerous algorithms for image resolution enhancement have been published since Tsai and Huang [1] first proposed their frequency-domain super-resolution approach. They differ in the observation model, reconstruction method, algorithm domain, and computational cost [2-10]. Most of the superresolution methods use the observation model where the observed low-resolution images result from warping, blurring, and subsampling operations performed on the highresolution image. They work in the spatial domain using maximum a posteriori (MAP) or project onto convex set (POCS) reconstruction technique, and are usually computationally expensive. A complete and comprehensive overview of super-resolution algorithms can be seen in $[11,12]$. On the other hand, super-resolution techniques have been successfully used in many applications including medical imaging, remote sensing, satellite imaging, high-definition television, surveillance systems, forensic science, video frame freezing, and printing. This paper explores a new application of super resolution image reconstruction in digital holography, and analyzes and evaluates the corresponding super-resolution algorithm in the context of digital holography. To my knowledge, this is the first time super-resolution is applied in enhancing the spatial resolution of digital holograms to overcome the problems caused by low-resolution sensor in digital holography.

Holography is a widely used technique for recording and reconstructing three-dimensional (3D) information of an object. In classical holography, photographic films are used to record holographic patterns and the reconstruction is performed optically. Recent advances in charge-coupled device (CCD) and computer technology have permitted replacing holographic films with CCD arrays and optical reconstruction with computer-driven numerical reconstruction. Digital holography is thus referred to as the technique that uses a CCD camera to record holographic patterns and performs the reconstruction numerically using a computer [13]. In comparison with classical optical holography, digital holography has the major advantage that it eliminates the need of wet chemical development processing and mechanical focusing and, thus, leads to much faster and flexible holographic processing. In addition, both the intensity and phase of the object can be retrieved from digital holograms. Accordingly, digital holography has been successfully applied in 3D information processing, microscopy, particle analysis, deformation studies, and surface contouring. 


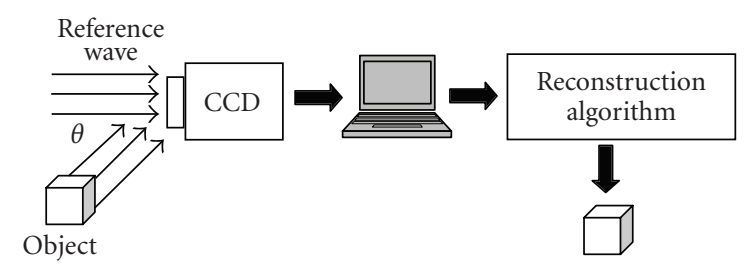

Figure 1: Digital holography.

Digital holography has been limited to record small objects only. This is due to the low spatial resolution of commercially available CCD arrays. Although the CCD resolution increases constantly, it is still lower than photographic material by at least one order of magnitude. In order to obtain a good reconstructed image quality and measurement accuracy, the sampling theorem has to be satisfied during the digital recording of holograms. The low resolution of CCD limits the angle between the object and reference beams in holography to only a few degrees in order to meet the sampling requirement [14]. The hologram will be undersampled if the recording angle is larger than the maximum acceptable angle. As a result, only small objects can be recorded and the objects must be placed far away from the CCD camera. In order to record a larger object, additional optical lens system is normally needed to reduce the object size [15]. Optical bandlimiting may be highly undesirable in some applications since the object details will be lost. To overcome the abovementioned problem, super-resolution image reconstruction is proposed here to increase the resolution of holograms and eliminate the aliasing effects caused by undersampling. By doing so, larger objects can be recorded and the distance between the object and the hologram can be shortened.

The principle of digital holography and the problems caused by low resolution of imaging sensors are briefly described next. Then the super-resolution algorithm used for hologram resolution enhancement and the obtained results are presented.

\section{DIGITAL HOLOGRAPHY AND ITS RESOLUTION PROBLEM}

Holography has been widely used for 3D information recording and reconstruction. It records $3 \mathrm{D}$ objects in twodimensional (2D) interference images and retrieves the intensity and phase of the objects from the recorded holograms. Figure 1 shows the principle of digital holography. In the recording phase, the object is illuminated by laser and the diffracted object wave is interfered with a reference plane wave. There should be a small incidence angle $\theta$ between the reference wave and the object wave in order to retrieve the object wave in the later reconstruction phase. The resulting interference pattern is captured by a CCD array and then stored in an image processing system. Let $\mathbf{R}$ and $\mathbf{O}$ be the reference and object waves at the CCD plane, respectively. The intensity I recorded by the CCD is given by

$$
\mathbf{I}=|\mathbf{R}+\mathbf{O}|^{2}=|\mathbf{R}|^{2}+|\mathbf{O}|^{2}+\mathbf{R} * \mathbf{O}+\mathbf{R O} *,
$$

where the asterisk denotes the complex conjugation. I is sampled by the CCD camera to produce a $2 \mathrm{D}$ numerical array $I(m, n)$. In the reconstruction phase, the object wave is retrieved numerically in a compute by multiplying the recorded hologram image with the reference wave $\mathbf{R}$. The reconstructed wavefront in the hologram plane is obtained as

$$
\mathbf{U}=\mathbf{R I}=\mathbf{R}|\mathbf{R}|^{2}+\mathbf{R}|\mathbf{O}|^{2}+|\mathbf{R}|^{2} \mathbf{O}+\mathbf{R}^{2} \mathbf{O} *
$$

The first two terms of (2) create a zero-order diffraction, the third term produces a virtual image located at the initial position of the object, and the fourth produces a real image of the object on the other side of the hologram. The complex amplitude of the object wave can be calculated by the Fresnel transform of the digitized hologram [13], which is given as

$$
\begin{aligned}
& U(m, n)= \exp \left[-\frac{i \pi}{\lambda z}\left(m^{2} \Delta \xi^{2}+n^{2} \Delta \eta^{2}\right)\right] \\
& \times \sum_{k=0}^{M-1} \sum_{l=0}^{N-1} I(k, l) R(k, l) \\
& \quad \times \exp \left[-\frac{i \pi}{\lambda z}\left(k^{2} \Delta x^{2}+l^{2} \Delta y^{2}\right)\right] \\
& \quad \times \exp \left[i 2 \pi\left(\frac{k m}{M}+\frac{l n}{N}\right)\right]
\end{aligned}
$$

where $\Delta x, \Delta y$, and $\Delta \xi, \Delta \eta$ are the pixel sizes in the hologram plane and in the reconstructed image plane, respectively. $z$ is the distance between these two planes, $\lambda$ is the laser wavelength, and $M \times N$ is the size of the hologram. The fast Fourier transform (FFT) algorithm is usually used to calculate (3) since it can be considered as a discrete Fourier transform.

The sampling theorem has to be met during the digital recording of holograms in order to obtain a good reconstructed image quality and measurement accuracy. The maximum spatial frequency to be recorded is limited by the resolution of CCD. For off-axis digital holography, the angle $\theta$ between the reference and object waves must not exceed a maximum value given by

$$
\theta_{\max }=\arcsin \left(\frac{\lambda}{2 \Delta x}\right)
$$

which normally is just a few degrees even with small objects [14]. If $\theta>\theta_{\max }$, the sampling theorem is not fulfilled and the hologram is undersampled. Figure 2 gives an example to show the effect of aliasing caused by hologram undersampling. Figure 2(a) is the object to be recorded and Figure 2(b) is the recorded hologram with Nyquist sampling. From Figure 2(b), we can reconstruct the object perfectly as shown in Figure 2(d), where, the real and the virtual image of the object are separated. If we downsample Figure 2(b) by a factor of 2 in each axis to obtain an undersampled hologram as shown in Figure 2(c), the reconstructed object image from Figure 2(c) will exhibits aliasing, as shown in Figure 2(e). 


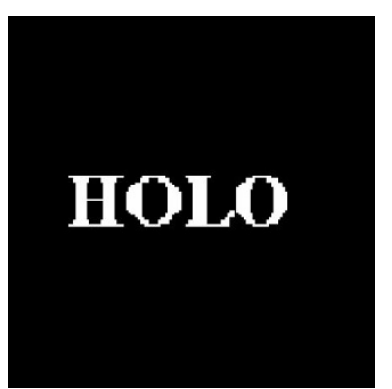

(a)

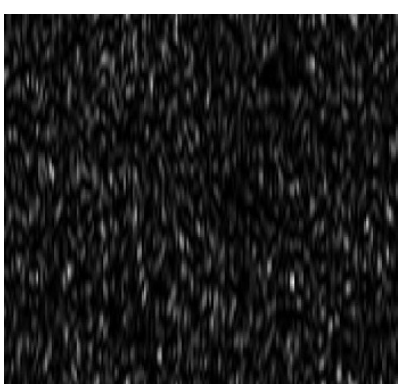

(b)

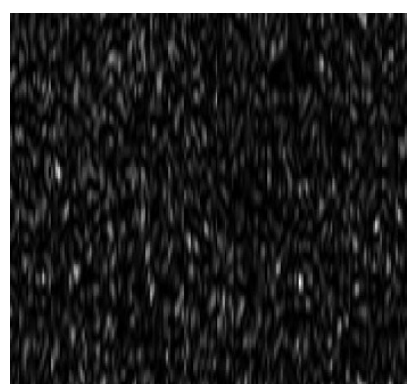

(c)

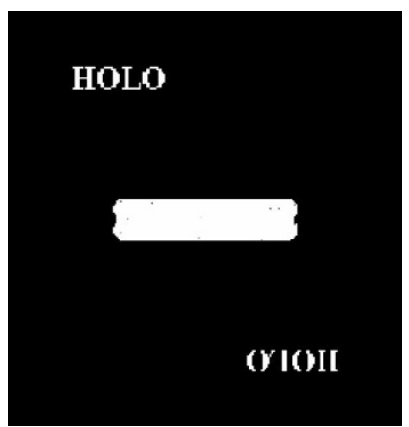

(d)

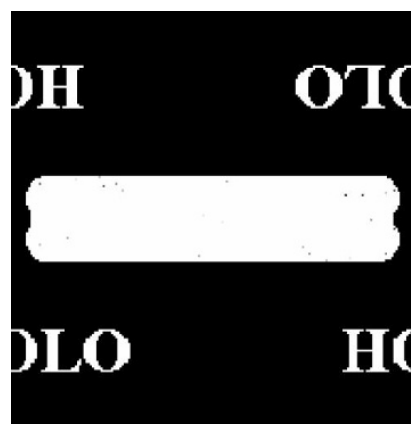

(e)

FIGURE 2: Demonstration of aliasing due to undersampling: (a) a binary object "HOLO;" (b) recorded hologram with Nyquist sampling; (c) undersampled hologram; (d) reconstructed image from (b); and (e) reconstructed object image from (c).

If we need to record a larger object, it must be placed far away from the camera due to the limitation of recording angle. This will degrade the quality of the hologram and may lose object details. Therefore it is highly desired to enhance the resolution of holograms to widen the angle for recording larger objects. To increase the resolution of images, the simplest method is to use interpolation. But interpolation methods are suboptimal and result in poor performance. Super-resolution has been demonstrated to have much better performance than interpolation, and thus is proposed here for enhancing the spatial resolution of holograms. We can capture several undersampled holograms and then use a super-resolution algorithm to reconstruct a high-resolution hologram such that the reconstructed high-resolution hologram can meet the Nyquist sampling rate.

\section{HIGH-RESOLUTION HOLOGRAM RECONSTRUCTION}

Super-resolution algorithms first need to have a suitable observation model to relate the original high-resolution image with the observed low-resolution images, and then employ some reconstruction technique worked in either the spatial or frequency domain. It is common to model each low-resolution image as a noisy, downsampled version of the shifted and blurred high-resolution image. Irani and Peleg [3] proposed an iterative back-projection method to reconstruct the high-resolution image by minimizing the error between the observed low-resolution images and the predicted low-resolution images that were obtained by projecting the high-resolution image estimate through the observation model. Hardie et al. [4] extended the back-projection method by employing a gradient descent and a conjugate gradient technique for minimizing regularized cost function, where, the subpixel motion was estimated using gradient method based on Taylor expansion. Cheeseman et al. [5] have developed a Bayesian estimation method with a prior knowledge of pixel neighbor correlation to improve the super-resolution algorithm. The success of super-resolution reconstruction largely depends on accurate motion estimation between low-resolution images. However, motion estimation with fractional pixel accuracy could be a difficult problem. Recently, some superresolution algorithms have been developed to incorporate inaccurate subpixel registration. $\mathrm{Ng}$ et al. $[6,7]$ considered the registration error by proposing a regularized total least squares method to minimize the error. Poletto and Nicolosi [8] proposed a super-resolution method that is insensitive to high uncertainties in the subpixel displacements in which images were subsequently acquired shifted by a fraction of a pixel with respect to each other. Lee and Kang [9] used channel adaptive regularization technique to minimize the effect of the registration error. Although most super-resolution methods can achieve good performance in reconstruction, they are usually computationally expensive. The time-consuming characteristics of iterative 


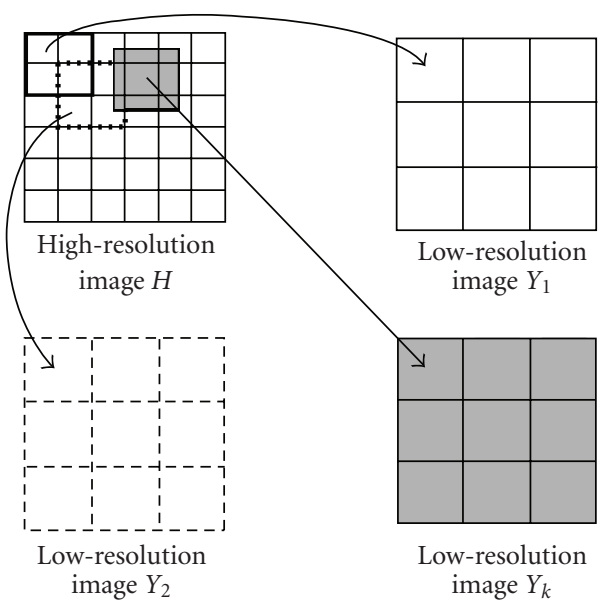

Figure 3: Super-resolution model.

super-resolution algorithms could exclude themselves from many practical applications. Nguyen et al. [10] proposed an efficient block circulant preconditioning technique to accelerate the convergence in reconstruction iteration. Elad and Hel-Or [2] demonstrated that fast noniterative calculation of image reconstruction is possible if blur is spaceinvariant, warps are pure translations, and additive noise is white.

For hologram image resolution enhancement, we can assume the observation model to be pure translational motion, universal space-invariant blur and decimation considering that holograms are typically captured inside a clean room and we can mount the CCD camera on a translational stage. Therefore, the fast noniterative super-resolution algorithm in [2] is well suited for enhancing hologram's resolution in realtime. The algorithm is briefly described below and the details can be seen in [2].

Assume we capture $P$ frames of low-resolution hologram images with a translating CCD camera and they are denoted as $Y_{k}$ for $k=1,2, \ldots, P$. Let $H$ be the original high-resolution hologram sampled at the Nyquist rate that we wish to estimate from those $P$ low-resolution holograms. Then $Y_{k}$ is related to the required super-resolution image $H$ through translation, blur, decimation, and additive noise, which can be represented using a matrix notation as

$$
Y_{k}=D B S_{k} H+V_{k}, \quad 1 \leq k \leq P,
$$

where $S_{k}$ is the matrix representing the translation performed on the hologram $H, B$ is the space-invariant blur matrix representing the camera's PSF, $D$ is the decimation operator resulting in $Y_{k}$, and $V_{k}$ is the Gaussian noise with zero-mean and auto correlation matrix $W_{k}=E\left[V_{k} V_{k}^{T}\right]$. Figure 3 illustrates the above super-resolution model. It shows the formation process of low-resolution images from a high-resolution image, where a square box (solid, dashed, or gray) represents a low-resolution detector. These boxes demonstrate how the high-resolution pixels contribute to a low-resolution pixel. The decimation matrix $D$ generates smaller size $(3 \times 3)$ low-resolution images from a large $(6 \times 6)$ high-resolution image. The blur matrix $B$ is implemented by summing the high-resolution pixels within the span of lowresolution detector (box). The translation matrix $S_{k}$ is indicated by the relative motion from the solid box to the dashed or the gray box.

In order to estimate $H$ based on the captured holograms $Y_{k}$, we need to know $D, B, S_{k}$, and $W_{k}$. $S_{k}$ can be read from the translational stage or obtained through motion estimation. The camera blur matrix $B$ can also be estimated or simplified to some simple known blur function. $D$ is the resolution ratio to obtain. The noise is assumed Gaussian and mutually independent for different images. Based on the above assumptions, it is shown in [2] that the maximumlikelihood estimation of $H$ can be obtained from the following least-squares equation:

$$
\hat{H}=\underset{H}{\arg \min }\left\{\sum_{k=1}^{P}\left[Y_{k}-D B S_{k} H\right]^{T} W_{k}^{-1}\left[Y_{k}-D B S_{k} H\right]\right\},
$$

or iterately from

$$
\hat{H}_{j+1}=\hat{H}_{j}+\mu \sum_{k=1}^{P} S_{k}^{T} B^{T} D^{T} W_{k}^{-1}\left[Y_{k}-D B S_{k} \hat{H}_{j}\right]
$$

where $\mu$ is a turning parameter. The algorithm can be further changed for fast noniterative calculation.

The procedure of using super-resolution for digital hologram enhancement is as follows. The interference pattern at the CCD plane is captured several times by a translating CCD camera. Each time the CCD camera is shifted to a different position so that a relative shift can be introduced between each frame acquisition. These shifted holograms are then processed using the above-described algorithm to obtain a high-resolution hologram. Finally, the 3D object is reconstructed from the resolution enhanced hologram.

\section{RESULTS}

Both simulated and real hologram images were used to demonstrate the feasibility of the proposed method. As in [2], the unknown PSF was assumed to be a 2D separable kernel constructed from the $1 \mathrm{D}$ blur $[0.25,0.5,0.25]$. The low-resolution hologram images were created from a known high-resolution hologram, and so motion vectors were known. The iteration algorithm in (7) was implemented and used for testing where $\mu=0.1$.

The synthetic $400 \times 400$ hologram in Figure 2(b) was downsampled to create four $200 \times 200$ undersampled low-resolution holograms by a factor of 2 at each axis. The reference low-resolution hologram was obtained by 


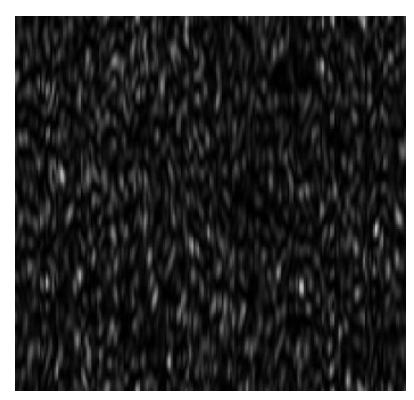

(a)

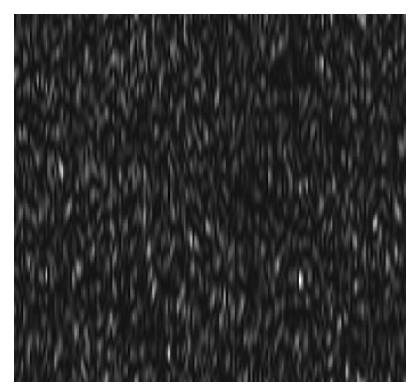

(c)

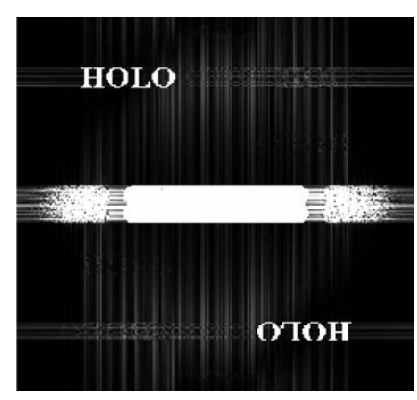

(b)

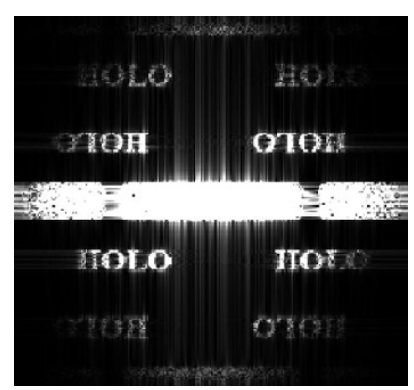

(d)

FIGURE 4: Results for simulated holograms: (a) super-resolution enhanced high-resolution hologram; (b) reconstructed object image from (a); (c) bilinearly interpolated hologram; and (d) reconstructed object image from (c).

decimation without shifting the high-resolution hologram, and the rest three low-resolution holograms were obtained by shifting the high-resolution hologram horizontally, vertically, and diagonally, respectively, by one pixel before decimation. One of the undersampled hologram used has been shown in Figure 2(c). A high-resolution reconstructed hologram was obtained, as shown in Figure 4(a), after applying the super-resolution algorithm to these four undersampled holograms. The corresponding reconstructed object from Figure 4(a) is shown in Figure 4(b). It is seen that aliasing has been eliminated. For comparison, Figures 4(c) and 4(d) provide the bilinearly interpolated hologram and the corresponding reconstructed object image, respectively.

Next a real $1024 \times 1024$ hologram shown in Figure 5(a) was used to test the super-resolution reconstruction for digital holography. This hologram was perfectly sampled. Figure 5(b) shows its holographical reconstruction, which gives the object recorded. Again, four undersampled low-resolution hologram images were created from the high-resolution hologram using the same parameters as the synthetic hologram. Figure 5(c) shows one of the downsampled holograms. Figure 5(d) is the object reconstruction result when this undersampled hologram was directly used. It is seen that the object is totally blurred. The result of using bilinear interpolation for resolution enhancement is presented in Figure 5(e). Figure 5(f) is the corresponding reconstructed object image, which shows that bilinear interpolation-based enhancement fails to retrieve the object. The high-resolution hologram using the super-resolution reconstruction was obtained as shown in Figure 5(g), and the corresponding reconstructed object is given in Figure 5(h). It yields much better performance than the interpolation method.

\section{CONCLUSIONS}

Low resolution of imaging sensors has been one of the main problems in digital holography, which produces undersampled holograms in recording relatively larger objects. Superresolution image reconstruction has been successfully applied to enhance the resolution of digital holograms by combining several shifted undersampled holograms. This makes the recording of large objects using short object-to-hologram distance become possible. The effectiveness of the proposed method has been verified by practical results from real and simulated holograms. The intension of this paper is to study the feasibility of using super-resolution in digital holography and provide the preliminary results, but not to find the best super-resolution algorithm for it. It is noted that the reconstructed high-resolution hologram is not identical to the original high-resolution hologram. This is not a big problem for $3 \mathrm{D}$ visualization, but it may limit the application in measurement where precision is a concern. Further investigation is thus required before it can be applied in practice. 


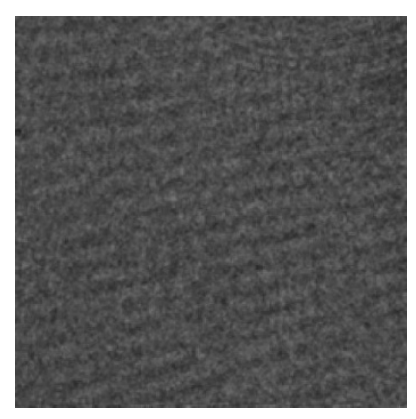

(a)

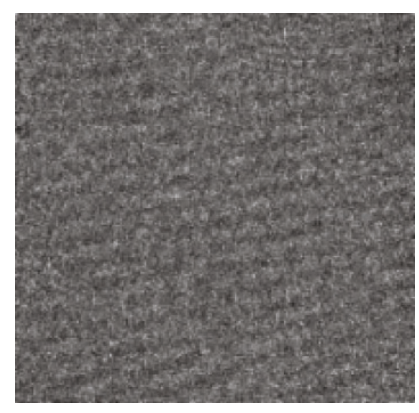

(c)

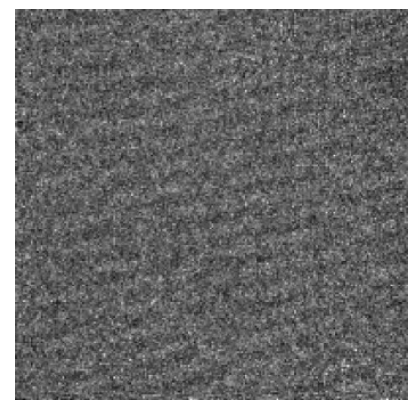

(e)

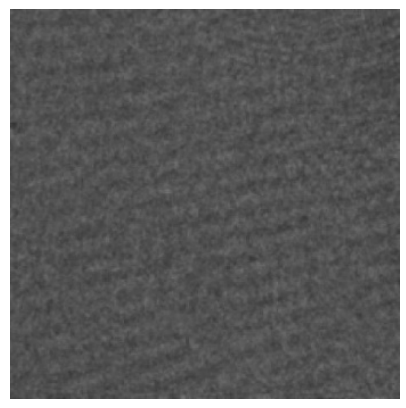

(g)

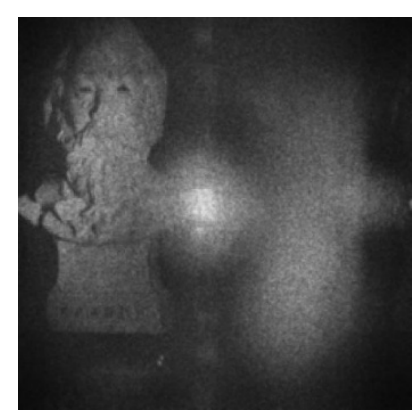

(b)

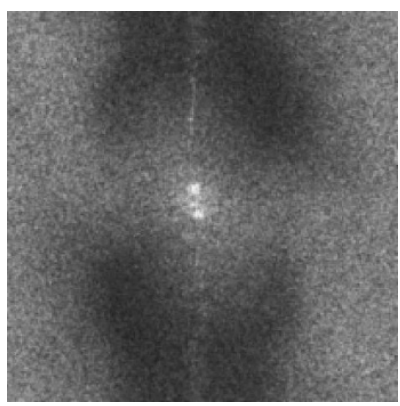

(d)

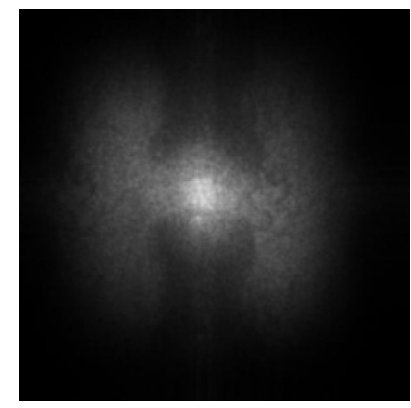

(f)

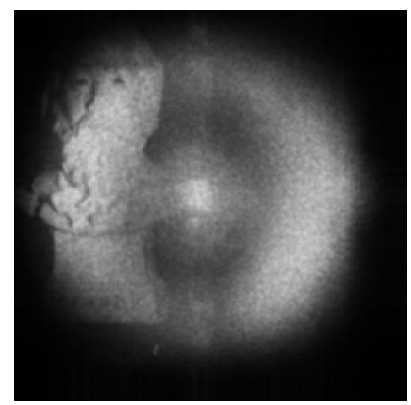

(h)

FIGURE 5: Results for real holograms: (a) original hologram meeting sampling rate (courtesy of Oystein Skotheim and Vegard L. Tuft, Norwegian University of Science and Technology, Norway); (b) reconstructed object image from (a); (c) one of the undersampled holograms; (d) reconstructed object image from (c); (e) bilinearly interpolated hologram; (f) reconstructed object image from (e); (g) super-resolution enhanced high-resolution hologram; and $(\mathrm{h})$ reconstructed object image from $(\mathrm{g})$. 


\section{ACKNOWLEDGMENT}

This work was supported by Grant 60092-34 35 from The City University of New York PSC-CUNY, Research Award Program.

\section{REFERENCES}

[1] R. Y. Tsai and T. S. Huang, "Multi-frame image restoration and registration," in Advances in Computer Vision and Image Processing, vol. 1, chapter 7, pp. 317-339, JAI Press, Greenwich, Conn, USA, 1984.

[2] M. Elad and Y. Hel-Or, "A fast super-resolution reconstruction algorithm for pure translational motion and common spaceinvariant blur," IEEE Transactions on Image Processing, vol. 10, no. 8, pp. 1187-1193, 2001.

[3] M. Irani and S. Peleg, "Improving resolution by image registration," CVGIP: Graphical Models and Image Processing, vol. 53, no. 3, pp. 231-239, 1991.

[4] R. C. Hardie, K. J. Barnard, J. G. Bognar, E. E. Armstrong, and E. A. Watson, "High-resolution image reconstruction from a sequence of rotated and translated frames and its application to an infrared imaging system," Optical Engineering, vol. 37, no. 1, pp. 247-260, 1998.

[5] P. Cheeseman, B. Kanefsky, R. Kraft, J. Stutz, and R. Hanson, "Super-resolved surface reconstruction from multiple images," in Maximum Entropy and Bayesian Methods, G. R. Heidbreder, Ed., pp. 293-308, Kluwer Academic, Dordrecht, the Netherlands, 1996.

[6] M. K. Ng, J. Koo, and N. K. Bose, "Constrained total least squares computations for high-resolution image reconstruction with multisensors," Journal of Imaging Science and Technology, vol. 12, no. 1, pp. 35-42, 2002.

[7] M. K. Ng and N. K. Bose, "Mathematical analysis of superresolution methodology," IEEE Signal Processing Magazine, vol. 20, no. 3, pp. 62-74, 2003.

[8] L. Poletto and P. Nicolosi, "Enhancing the spatial resolution of a two-dimensional discrete array detector," Optical Engineering, vol. 38, no. 10, pp. 1748-1757, 1999.

[9] E. S. Lee and M. G. Kang, "Regularized adaptive highresolution image reconstruction considering inaccurate subpixel registration," IEEE Transactions on Image Processing, vol. 12, no. 7, pp. 826-837, 2003.

[10] N. Nguyen, P. Milanfar, and G. Golub, "A computationally efficient super-resolution image reconstruction algorithm," IEEE Transactions on Image Processing, vol. 10, no. 4, pp. 573-583, 2001.

[11] S. C. Park, M. K. Park, and M. G. Kang, "Super-resolution image reconstruction: a technical overview," IEEE Signal Processing Magazine, vol. 20, no. 3, pp. 21-36, 2003.

[12] S. Borman and R. L. Stevenson, "Spatial resolution enhancement of low-resolution image sequences: a comprehensive review with directions for future research," Tech. Rep., Laboratory for Image and Signal Analysis (LISA), University of Notre Dame, Notre Dame, Pa, USA, 1998.

[13] U. Schnars and W. P. O. Jüptner, "Direct recording of holograms by a CCD target and numerical reconstruction," Applied Optics, vol. 33, no. 2, pp. 179-181, 1994.

[14] U. Schnars and W. P. O. Jüptner, "Digital recording and reconstruction of holograms in hologram interferometry and shearography," Applied Optics, vol. 33, no. 20, pp. 4373-4377, 1994.
[15] U. Schnars, T. M. Kreis, and W. P. O. Jüptner, "Digital recording and numerical reconstruction of holograms: reduction of the spatial frequency spectrum," Optical Engineering, vol. 35, no. 4, pp. 977-982, 1996.

Shuqun Zhang received his $\mathrm{Ph.D}$. degree in electrical engineering from the University of Dayton in 1999, and his M.S. and B.S. degrees in electrical engineering from Xiamen University, China in 1994 and 1991, respectively. He is currently an Assistant Professor in the Department of Computer Science, College of Staten Island, City University of New York. His current research interests include image processing, pattern recognition, and optical information processing. He is a Member of the IEEE, the OSA, and the SPIE.

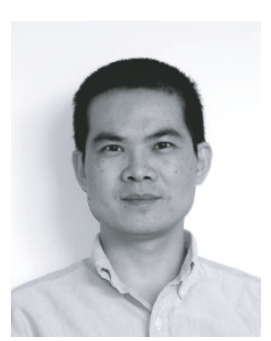

